\title{
THE HOMOLOGY OF TWISTED CARTESIAN PRODUCTS
}

\author{
BY \\ R. H. SZCZARBA(1)
}

Introduction. In a recent paper [2], E. H. Brown introduced the notion of a twisted tensor product. Briefly, the definition is as follows.

Let $K$ be a D.G.A. (differential, graded, augmented) coalgebra, $A$ a D.G.A. algebra, and $M$ a D.G.A. $A$-module. The twisted tensor product $K_{\phi} \otimes M$ of $K$ with $M$ is, except for the differential, the usual tensor product. The differential on $K_{\phi} \otimes M$ is modified using a twisting cochain $\phi$ in $\operatorname{Hom}(K, A)$. Now suppose $p: E \rightarrow X$ is a fiber space (essentially of the Hurewicz type) with fiber $F$. Then $C(X)$ is a D.G.A. coalgebra, $C(\Omega X)$ a D.G.A. algebra, and $C(F)$ a D.G.A. $C(\Omega X)$-module. Brown's main theorem states that there is a twisting cochain $\phi$ in $\operatorname{Hom}(C(X), C(\Omega X))$ and a chain equivalence $\psi: C(X)_{\phi} \otimes C(F) \rightarrow C(E)$.

In another recent paper [1], Barratt, Gugenheim, and Moore define the twisted cartesian product of two simplicial sets (semi-simplicial complexes). If $X$ and $F$ are simplicial sets and $G$ is a simplicial group acting on $F$, the twisted cartesian product of $X$ and $F, X \times{ }_{\tau} F$, coincides with the usual cartesian product except that the initial face is modified in terms of a twisting function $\tau: X \rightarrow G$. It is proved in [1] that any simplicial fiber space $p: E \rightarrow X$ with fiber $F$ can, for the purposes of algebraic topology, be replaced by a twisted cartesian product $X \times_{\tau} F$. (The group $G$ and the twisting function $\tau: X \rightarrow G$ are shown to exist.)

Considering these two results, one might expect that an analogue of Brown's theorem could be proved for twisted cartesian products, explicitly defining the twisting cochain in terms of the twisting function $\left({ }^{2}\right)$. This is in fact done in Part I of this paper.

In Part II, the explicit form of the twisting cochain is used to investigate fiber bundles over spheres. The homology and cohomology Wang sequences are derived and some partial results obtained describing the behavior of the maps in the cohomology Wang sequence with respect to cup products.

I would like to express my gratitude to Professor Saunders MacLane for his patient assistance and encouragement during the preparation of this

Received by the editors January 19, 1961.

(1) The results of this paper are taken from the author's Ph.D. thesis submitted to the Mathematics Department of the University of Chicago in September, 1960.

(2) V. K. A. M. Gugenheim in [3] does prove Brown's theorem for twisted cartesian products without giving an explicit definition of the twisting cochain. 
paper. I would also like to thank V. K. A. M. Gugenheim for suggesting simplifications that appear in the proof of Theorem 2.4.

\section{PART I}

1. Preliminaries. In what follows, $\Lambda$ will denote a fixed commutative ring with a unit. All algebras, coalgebras, tensor products, etc. will be taken over $\Lambda$ unless explicitly stated to the contrary.

Let $A$ be a D.G.A. algebra with product $\pi: A \otimes A \rightarrow A$ and $K$ a D.G.A. coalgebra with coproduct $\Delta: K \rightarrow K \otimes K$. A twisting cochain is a cochain $\phi=\sum \phi_{j}$ in $C^{*}(K ; A)$ such that

$$
\begin{gathered}
\phi_{q} \in C^{q}(K ; A), \quad \phi_{0}=0, \quad \phi_{q} K_{q} \subset A_{q-1}, \\
\epsilon \phi_{1}=0 \quad \text { and } \quad \partial \phi_{q}=\phi_{q-1} \partial-\sum_{k=1}^{q-1}(-1)^{k} \phi_{k} \cup \phi_{q-k},
\end{gathered}
$$

where the cup product $\phi_{k} \cup \phi_{q-k}$ is the composite

$$
K \stackrel{\Delta}{\rightarrow} K \otimes K \stackrel{\phi_{k} \otimes \phi_{q-k}}{\rightarrow} A \otimes A \stackrel{\pi}{\rightarrow} A .
$$

Suppose $M$ is a D.G.A. $A$-module with product $\mu: A \otimes M \rightarrow M$. The twisted tensor product of $K$ and $M$ with respect to the twisting cochain $\phi$ in $C^{*}(K ; A)$ is the D.G.A. module $K_{\phi} \otimes M$ defined by

$$
\begin{aligned}
&\left(K_{\phi} \otimes M\right)_{q}=\sum_{i+j=q} K_{i} \otimes M_{j}, \\
& \epsilon(k \otimes m)=\epsilon(k) \cdot \epsilon(m), \\
& \partial_{\phi}(k \otimes m)=(\partial k) \otimes m+(-1)^{q}[k \otimes \partial m+k \otimes m \cap \phi], \quad k \in K_{q} .
\end{aligned}
$$

The cap product $k \otimes m \cap \phi \in K \otimes M$ is defined to be the value of the composite

$$
K \otimes M \stackrel{\Delta \otimes 1}{\longrightarrow} K \otimes K \otimes M \stackrel{1 \otimes \phi \otimes 1}{\longrightarrow} K \otimes A \otimes M \stackrel{1 \otimes \mu}{\rightarrow} K \otimes M
$$
on $k \otimes m$.

Let $X$ be a simplicial set (=semi-simplicial complex) and $G$ a simplicial group [9]. A twisting function is a map $\tau: X \rightarrow G$ satisfying

$$
\begin{aligned}
\tau\left(X_{q}\right) & \subset G_{q-1}, \\
d_{0} \tau(x) & =\tau\left(d_{0} x\right)^{-1} \cdot \tau\left(d_{1} x\right), \\
d_{i} \tau(x) & =\tau\left(d_{i+1} x\right), \\
s_{i} \tau(x) & =\tau\left(s_{i+1} x\right), \\
\tau\left(s_{0} x\right) & =\text { identity. }
\end{aligned}
$$$$
i \geqq 0 \text {, }
$$

In these equations, $d_{i}$ and $s_{i}$ denote the face and degeneracy maps. 
Now, suppose $G$ acts on a simplicial set $F$. The twisted cartesian product of $X$ with $F$ relative to $\tau$ is the simplicial set $X X_{\tau} F$ defined by

$$
\begin{aligned}
\left(X X_{\tau} F\right)_{q} & =X_{q} \times F_{q}, \\
d_{0}(x, y) & =\left(d_{0} x, \tau(x) \cdot d_{0} y\right), \\
d_{i}(x, y) & =\left(d_{i} x, d_{i} y\right), \\
s_{i}(x, y) & =\left(s_{i} x, s_{i} y\right),
\end{aligned}
$$

A simplicial operator $D$ of type $(p, q), p$ and $q$ nonnegative integers, is a collection of maps $D(X): X_{p} \rightarrow X_{q}$, one for each simplicial set $X$, natural with respect to simplicial maps. The integer $q-p$ is called the degree of $D$.

Theorem 1.1. Any simplicial operator $D$ of type $(p, q)$ can be written uniquely as

$$
D=s_{i_{r}} \cdots s_{i_{1}} d_{j_{s}} \cdots d_{j_{1}}
$$

where $r-s=q-p, i_{r}>i_{r-1}>\cdots>i_{1}$, and $j_{s}<j_{s-1}<\cdots<j_{1} \leqq p$.

Proof. (See $[4$, p. 59]). Suppose $D$ is a simplicial operator of type $(p, q)$ and $X$ a simplicial set with $x \in X_{p}$. Let $\Delta^{p}$ be the simplicial set representing the standard $p$-simplex and $\kappa_{p} \in \Delta^{p}$ the nondegenerate $p$-simplex. As is well known, there is a unique simplicial map $f: \Delta^{p} \rightarrow X$ with $f\left(\kappa_{p}\right)=x$. Writing $D\left(\Delta^{p}\right) \kappa_{p}=\rho \kappa_{p}$, where $\rho=s_{i_{r}} \cdots s_{i_{1}} d_{j_{s}} \cdots d_{j_{1}}, i_{r}>\cdots>i_{1}, j_{s} \cdots<j_{1} \leqq p$, and $r-s=q-p$ (any simplex of $\Delta^{p}$ can be written in this form), we see that

$$
D(X) x=D(X) f\left(\kappa_{p}\right)=f D\left(\Delta^{\rho}\right) \kappa_{p}=f\left(\rho \kappa_{p}\right)=\rho f\left(\kappa_{p}\right)=\rho x .
$$

Thus we see that, for any simplicial operator $D$ of type $(p, q)$, we have defined a family of simplicial operators of type $(p+k, q+k), k \geqq 0$, given by (1.2). We will refer to this family of simplicial operators as a simplicial operator of degree $q-p$ and initial dimension $p$ or simply as a simplicial operator.

For any simplicial operator $D=s_{i_{r}} \cdots s_{i_{1}} d_{j_{s}} \cdots d_{j_{1}}$ of degree $r-s$ and initial dimension $p$, we define a derived simplicial operator $D^{\prime}$ of degree $r-s$ and initial dimension $p+1$ by

$$
D^{\prime}=s_{i_{\mathrm{r}}+1} \cdots s_{i_{1+1}} d_{j_{\bullet}+1} \cdots d_{j_{1}+1} .
$$

Leмma 1.2. Let $D$ be a simplicial operator. Then $d_{0} D^{\prime}=D d_{0}$. If we assume $D$ contains no $d_{0}$, then $s_{0} D=D^{\prime} s_{0}$ and $D \tau(x)=\tau\left(D^{\prime} x\right)$ for any twisting function $\tau$.

The first two statements of the lemma are proved in $[4$, p. 60]. The third follows from (1.1).

For any two simplicial sets $X$ and $Y$, Eilenberg and MacLane [4; 5] define chain maps $\left({ }^{3}\right) f: C(X \times Y) \rightarrow C(X) \otimes C(Y)$ and $\nabla: C(X) \otimes C(Y) \rightarrow C(X \times Y)$ by the formulas

( $\left.{ }^{3}\right) C(X)$ denotes the normalized chain complex defined in [4]. 


$$
\begin{aligned}
& f(x \times y)=\sum_{i=0}^{p} d_{i+1}^{p-i} x \otimes d_{0}^{i} y, \\
& \nabla(x \otimes z)=\sum(-1)^{\operatorname{sgn}(\mu, \nu)} s_{\mu} x \times s_{\nu} z,
\end{aligned}
$$

where $x, y$ in $X_{p}, z$ in $Y_{q}$ and the second sum is over all $(q, p)$ shuffles $(\mu, \nu)$. They also define a chain homotopy $H: C(X \times Y) \rightarrow C(X \times Y)$ and prove that $f \nabla=$ identity and $\partial H+H \partial=\nabla f$-identity. The results of the present paper depend heavily on these facts.

2. The main theorems. In this section, we state the principal results of the paper.

Theorem 2.1. Let $X \times_{\tau} F$ be a twisted cartesian product with group $G$. For each pair of integers $(i, n), n \geqq 1,1 \leqq i \leqq(n-1)$ !, there exists a sequence of simplicial operators

$$
D_{0, i}^{n}, D_{1, i}^{n}, \cdots, D_{n-1, i}^{n},
$$

$D_{j, i}^{n}$ of degree $j$ and initial dimension $n-j-1$, and a mapping $\epsilon: Z \times Z \rightarrow Z_{2}(Z$ the integers, $Z_{2}$ the integers mod 2) such that the functions $\Phi_{n}: C(X) \rightarrow C(G)$ defined by

$$
\Phi_{n}(x)= \begin{cases}\sum_{i=1}^{(n-1) !}(-1)^{\epsilon(i, n)} D_{0, i}^{n} \sigma(x) \cdot D_{1, i}^{n} \sigma\left(d_{0} x\right) \cdots D_{n-1, i}^{n} \sigma\left(d_{0}^{n-1} x\right), & q \geqq n, \\ 0, & a<n,\end{cases}
$$

$x \in C_{q}(X), \sigma(x)=(\tau(x))^{-1}$, satisfy the following.

$$
\begin{aligned}
\Phi_{n} C_{q}(X) & \subset C_{q-1}(G), \\
d_{0} \Phi_{n}(x) & =\sum_{k=1}^{n-1}(-1)^{k} \Phi_{n-1}\left(d_{k} x\right), \\
d_{k} \Phi_{n}(x) & =0, \\
d_{n-1} \Phi_{n}(x) & =\sum(-1)^{k+n+\operatorname{sgn}(\mu, \nu)} s_{\mu} \Phi_{k}\left(d_{k+1}^{n-k} x\right) \cdot s_{\nu} \Phi_{n-k}\left(d_{0}^{k} x\right), \quad n<k<n-1,
\end{aligned}
$$

The sum in (2.4) is over $k=1$ to $n-1$ and over all $(n-k-1, k-1)$ shuffles $(\mu, \nu)$.

The proof of this theorem will be given in the next section.

Again letting $X \times_{T} F$ be a twisted cartesian product with group $G$, it is well known that $C(X)$ is a D.G.A. coalgebra, $C(G)$ a D.G.A. algebra, and $C(F)$ a D.G.A. $C(G)$-module. Define a cochain $\phi=\sum \phi_{k}$ in $\operatorname{Hom}(C(X), C(G))$ by

$$
\begin{aligned}
& \phi_{0}=0, \\
& \phi_{1}=\Phi_{1} \mid C_{1}(X)-e_{0}, \quad e_{0} \text { the identity in } G_{0}, \\
& \phi_{q}=\Phi_{q} \mid C_{q}(X) .
\end{aligned}
$$


Clearly $\phi_{q} \in C^{q}(K, A), \phi_{q} C_{q}(X) \subset C_{q-1}(G), \epsilon \phi_{1}=0$ and, using (2.1), ․ (2.4), we see that, for $q>1$,

$$
\begin{aligned}
\partial \phi_{q}(x)= & d_{0} \phi_{q}(x)+(-1)^{q-1} d_{q-1} \phi_{q}(x) \\
= & \sum_{k=1}^{q-1}(-1)^{k} \phi_{q-1}\left(d_{k} x\right)-\sum_{i=1}^{q-1}(-1)^{\gamma} s_{\mu} \phi_{i}\left(d_{i+1}^{q-i} x\right) \cdot s_{\nu} \phi_{q-i}\left(d_{0}^{i} x\right) \\
& +\phi_{q-1}\left(d_{0} x\right)+(-1)^{q} \phi_{q-1}\left(d_{q} x\right) \\
= & \phi_{q-1}(\partial x)-\sum_{i=1}^{q-1}(T 1)^{i}\left(\phi_{i} \cup \phi_{q-i}\right)(x),
\end{aligned}
$$

where $\gamma=i+\operatorname{sgn}(\mu, \nu)$. Thus we have proved the following.

THEOREM 2.2. Let $X \times_{\tau} F$ be a twisted cartesian product with group $G$. Then there is a twisting cochain $\phi=\sum \phi_{j}$ in $\operatorname{Hom}(C(X), C(G))$ with $\phi_{1}(x)=\sigma(x)-e_{0}$.

Define a map $\psi: C(X)_{\phi} \otimes C(F) \rightarrow C(X \times, F)$ by

$$
\begin{array}{r}
\psi(x \otimes y)=(1 \times \mu) \nabla \sum_{i=1}^{p}(-1)^{\epsilon(i, p+1)+p} D_{0, i}^{p+1} x \times D_{1, i}^{p+1} \sigma(x) \cdots D_{p, i}^{p+1} \sigma\left(d_{0}^{p-1} x\right) \otimes y \\
x \in X_{p},
\end{array}
$$

where $\nabla: C(X \times G) \otimes C(F) \rightarrow C(X \times G \times F)$ is the Eilenberg-MacLane shuffle map and $\mu: G \times F \rightarrow G$.

THEOREM 2.3. $\psi \partial_{\phi}=\partial \psi$.

THEOREM 2.4. The map $\psi_{*}: H\left(C(X)_{\phi} \otimes C(F)\right) \rightarrow H\left(C\left(X \times_{\tau} F\right)\right)$ is an isomorphism.

The proofs of these theorems will be given in $\$ \S 4$ and 5 .

3. Proof of Theorem 2.1. The operators $D_{j, i}^{n}$ and the map $\epsilon$ are defined inductively as follows.

$$
\begin{aligned}
D_{0,1}^{1} & =\text { identity, } \\
D_{j, i+k(n-1) !}^{n+1}= & \left\{\begin{array}{lr}
D_{j, i}^{n^{\prime}} S_{0} d_{k-j}, \\
D_{j, i}^{n^{\prime}}, & 0 \leqq j<k, \\
D_{j-1, i}^{n^{\prime}}, S_{0}, & j=k,
\end{array}\right.
\end{aligned}
$$

and

$$
\begin{aligned}
\epsilon(1,1) & =0, \\
\epsilon(i+k(n-1) !, n+1) & =\epsilon(i, n)+k+1(\bmod 2), \\
\epsilon(i, n) & =0
\end{aligned}
$$


Before proving that the functions $\Phi_{n}$ have the required properties, we prove four lemmas.

Leмма 3.1. For any $n>0, D_{j, i}^{n}$ contains no $d_{0}$.

This lemma follows immediately from (3.1).

LEMMA 3.2. For any $n>1$,

$$
d_{0} D_{j, i+k(n-1) !}^{n+1}= \begin{cases}D_{j, i}^{n} d_{k-j}, & 0 \leqq j \leqq k, \\ D_{j-1, i}^{n}, & k<j \leqq n,\end{cases}
$$

where $1 \leqq i \leqq(n-1) !, 0 \leqq k \leqq n-1$.

This lemma follows easily from (3.1) and Lemma 1.2.

Lemma 3.3. For each pair of integers $(i, n), n>1,1 \leqq i \leqq(n-1)$ !, there exists a unique quadruple $(r, s, t,(\mu, \nu)), r, s, t$ integers with $1 \leqq r<n, 1 \leqq s$ $\leqq(r-1)$ !, and $1 \leqq t \leqq(n-r-1) !,(\mu, \nu) a(n-r-1, r-1)$ shuffle such that for all $j, 0 \leqq j<n$,

$$
d_{n-1} D_{j, i}^{n}= \begin{cases}s_{\mu} D_{j, 8}^{r} d_{r-j}^{n-r}, & 0 \leqq j<r, \\ s_{\nu} D_{j-r, t}^{n-r}, & r \leqq j<n,\end{cases}
$$

and

$$
\epsilon(i, n)=\epsilon(s, r)+\epsilon(t, n-r)+\operatorname{sgn}(\mu, \nu)+r+n \bmod 2 .
$$

Conversely, for each $n>1$ and quadruple $(r, s, t,(\mu, \nu))$ as above, there is a pair $(i, n)$ such that (3.3) and (3.4) hold.

Proof. The lemma is easily verified for $n=2$; suppose it true for $n \leqq N$, $N>2$. Applying $d_{N}$ to (3.1) with $n=N$, we express $d_{N} D^{N+1}$ in terms of $d_{N} D^{N^{\prime}}$. Passing to derived operators in (3.3) (again with $n=N$ ), we express $d_{N} D^{N^{\prime}}$ in terms of $D^{r^{\prime}}$ and $D^{N-r^{\prime}}$. Supposing $k<r$ and combining, we have

$$
d_{N} D_{j, i+k(N-1) !}^{N+1}=\left\{\begin{array}{lr}
s_{\mu}^{\prime} D_{j, 8}^{r^{\prime}} d_{r-j+1}^{N-r} s_{0} d_{k-j}, & 0 \leqq j<k, \\
s_{\mu}^{\prime} D_{j, 8}^{r^{\prime}} d_{r-j+1}^{N-r}, & j=k, \\
s_{\mu}^{\prime} D_{j-1,8}^{r^{\prime}} d_{r-j+2}^{N-r} s_{0}, & k<j<r+1, \\
s_{\nu}^{\prime} D_{j-r-1, t}^{N-r^{\prime}}, & r+1 \leqq j \leqq N .
\end{array}\right.
$$

Using Lemma 1.2, the basic identities relating the face and degeneracy maps, and (3.1) with $n=r$, we can rewrite (3.4) as

$$
d_{N} D_{j, i+k(N-1) !}^{N+1}=\left\{\begin{array}{lc}
s_{\mu}^{\prime} D_{j, s+k(r-1) j}^{r+1} d_{r+1-j}^{N-r}, & 0 \leqq j<r+1, \\
s_{\nu}^{\prime} s_{0} D_{j-r-1, t}^{N-r}, & r+1 \leqq j \leqq N .
\end{array}\right.
$$


Letting $s_{\bar{\mu}}=s_{\mu}^{\prime}, s_{\bar{\nu}}=s_{\nu}^{\prime} s_{0},(3.5)$ takes the form of (3.3).

Now, noting that $\operatorname{sgn}(\mu, \nu)=\operatorname{sgn}(\bar{\mu}, \bar{\nu})$, we have

$$
\begin{aligned}
\epsilon(i+k(N-1) !, N+1)= & \epsilon(i, N)+k+1 \\
= & \epsilon(s, r)+\epsilon(t, N-r)+\operatorname{sgn}(\bar{\mu}, \bar{\nu})+N+k+r+1 \\
= & \epsilon(s+k(r-1) !, r+1)+\epsilon(t, N-r) \\
& +\operatorname{sgn}(\bar{\mu}, \bar{\nu})+N+1+r+1,
\end{aligned}
$$

all equations mod 2. Thus, for $k<r$, the quadruple $(r+1, s+k(r-1) !, t,(\bar{\mu}, \bar{\nu}))$ corresponds to the pair $(i+k(N-1) !, N+1)$ as required in the lemma.

A similar argument shows that, for $k \geqq r$, the quadruple $(r, s, t+(k-r)(N-r-1) !,(\tilde{\mu}, \tilde{\nu}))$ corresponds to the pair $(i+k(N-1) !, N+1)$ where

$$
(\tilde{\mu}, \tilde{\nu})=\left(\mu_{N-r-1}+1, \cdots, \mu_{1}+1,0 ; \quad \nu_{r-1}+1, \cdots, \nu_{1}+1\right) .
$$

We now prove uniqueness. Suppose the quadruple $(r, s, t,(\mu, \nu))$ corresponds to the pair $(i+k(N-1) !, N+1)$ as required and suppose $k<r$. Using (3.1), we have

$$
\begin{array}{rlrl}
d_{N} D_{j, i}^{N^{\prime}} s_{0} d_{k-j} & =s_{\mu} D_{j, s}^{r} d_{r-j}^{N+1-r}, & 0 \leqq j<k \\
d_{N} D_{j, i}^{N^{\prime}}=s_{\mu} D_{j, 8}^{r} d_{r-j}^{N+1-r}, & j=k, \\
d_{N} D_{j-1, i}^{N^{\prime}} & =s_{\mu} D_{j, s}^{r} d_{r-j}^{N+1-r}, & k<j<r, \\
d_{N} D_{j-1, i}^{N^{\prime}} s_{0} & =s_{v} D_{j-r, t}^{N+1-r}, & r \leqq j \leqq N .
\end{array}
$$

Note that, since $d_{N} D_{j, i}^{N^{\prime}}$ contains no $s_{0}$, by the second equation of (3.6) we conclude that $s_{\mu}$ contains no $s_{0}$.

Write $s=p+q(r-2) !, 1 \leqq p \leqq(r-1) !, 0 \leqq q \leqq r-1$ and suppose $q>k$. Then, using (3.1) and (3.6), we have

$$
d_{N} D_{k, i}^{N^{\prime}}=s_{\mu} D_{k, p}^{r-1^{\prime}} s_{0} d_{q-j} d_{r-j}^{N+1-j} .
$$

By Lemma 3.1, $D_{k, p}^{r-1}$ contains no $d_{0}$ so $D_{k, p}^{r-1^{\prime}}$ contains no $d_{1}$. Thus, the right side of (3.7) contains an $s_{0}$ which is impossible since the left side does not. Supposing $q<k$ leads to a similar contradiction so $q=k$.

Let $s_{\mu}=s_{\bar{\mu}}^{\prime}$ and $s_{\nu}=s_{\bar{\nu}}^{\prime} s_{0}$. Applying Lemma 1.2, we can rewrite (3.6) as

$$
\begin{array}{rlrl}
\left(d_{N-1} D_{j, i}^{N}\right)^{\prime} s_{0} d_{k-j} & =\left(s_{\bar{\mu}} D_{j, p}^{r-1} d_{r-j-1}^{N+1-r}\right)^{\prime} s_{0} d_{k-j}, & 0 \leqq j<k, \\
\left(d_{N-1} D_{j, i}^{N}\right)^{\prime}=\left(s_{\bar{\mu}} D_{j, p}^{r-1} d_{r-j-1}^{N+1-r}\right)^{\prime}, & j=k, \\
\left(d_{N-1} D_{j, i}^{N}\right)^{\prime} s_{0}=\left(s_{\bar{\mu}} D_{j-1, p}^{r-1} d_{r-j}^{N+1-r}\right)^{\prime} s_{0}, & k<j<r, \\
\left(d_{N-1} D_{j, i}^{N}\right)^{\prime} s_{0}=\left(s_{\bar{\nu}} D_{j-r, t}^{N+1-r}\right)^{\prime} s_{0}, & r \leqq j<N+1 .
\end{array}
$$


As is easily seen, this implies that

$$
d_{N-1} D_{j, i}^{N}=\left\{\begin{array}{lc}
s \bar{\mu} D_{j, p}^{r-1} d_{r-1-j}^{N-(r-1)}, & 0 \leqq j<r-1, \\
s \bar{\nu} D_{j-(r-1), t}^{N-(r-1)}, & r-1 \leqq j \leqq N .
\end{array}\right.
$$

If $k \geqq r$, a similar result holds. A simple induction argument now shows that the quadruple corresponding to a pair $(i, n)$ is unique.

To prove that, for each quadruple $(r, s, t,(\mu, \nu))$, of the prescribed type, there is a pair $(i, n)$ such that (3.3) holds, we essentially reverse the argument above. Suppose it true for $n \leqq N$ and let $(r, s, t,(\mu, \nu))$ be a quadruple with $1 \leqq r \leqq N, 1 \leqq s \leqq(r-1) !, 1 \leqq t \leqq(N-r)$ !, and $(\mu, \nu)$ a $(N-r, r-1)$ shuffle. Suppose $0 \in \nu$ and define $(\bar{\mu}, \bar{\nu})\left(\mu_{N-r}-1, \cdots, \mu_{1}-1 ; \nu_{r-1}-1, \cdots, \nu_{2}-1\right)$. This is clearly a $(N-r, r-2)$ shuffle. Write $s=p+k(r-2) !$ and, by induction, choose a pair $(i, N)$ such that (3.3) holds for $(r-1, p, t,(\bar{\mu}, \bar{\nu}))$. Then (3.8) and therefore (3.6) holds. From this it is easily seen that the pair $(i+k(N-1) !, N+1)$ corresponds to the quadruple $(r, s, t,(\mu, \nu))$. A similar argument holds for $0 \in \mu$.

Lemma 3.4. Let $P_{n}=\{1,2, \cdots,(n-1) !\}, n>0$. Then, for each pair of integers $(m, n), n>2,0<m<n-1$, there is a partition of $P_{n}$ into pairs $\left(i_{1}, i_{2}\right)$ such that $d_{m} D_{j, i_{1}}^{n}=d_{m} D_{j, i_{2}}^{n}, 0 \leqq j<n$, and $\epsilon\left(i_{1}, n\right)=1+\epsilon\left(i_{2}, n\right) \bmod 2$.

Proof. The lemma is easily verified for small values of $n$. Suppose it true for $n \leqq N$ and consider a pair $(m, N+1), 0<m<N$.

CASE 1. $1<m<N$. By induction, corresponding to the pair $(m-1, N)$, there is a partition of $P_{N}$ into pairs $\left(i_{1}, i_{2}\right)$ satisfying the conditions of the lemma. For each of these pairs $\left(i_{1}, i_{2}\right)$ and each integer $k, 0 \leqq k<N$, consider the pairs $\left(i_{1}+k(N-1) !, i_{2}+k(N-1) !\right)$. These clearly partition $P_{N+1}$ and, using (3.1) and (3.2), we see easily that this partition is as required.

CASE 2. $m=1$. Consider a pair $(i, N+1)$ and write $i=j+r(N-2)$ ! $+k(N-1) !, 1 \leqq j \leqq(N-2) !, 0 \leqq r \leqq N-2,0 \leqq k \leqq N-1$. Then, applying (3.1) twice, we see that the pairs $(j+r(N-2) !+k(N-1) !, j+(k-1)(N-2)$ ! $+r(N-1) !)$ where $0 \leqq r<k \leqq N-1$ partition $P_{N+1}$ as required.

We now complete the proof of Theorem 2.1. The verification of (2.1) is trivial as are the verifications (2.2), (2.3), and (2.4) when $q<n, x \in C_{q}(X)$. For $q \geqq n,(2.3)$ follows directly from Lemma 3.4 and (2.4) from Lemma 3.3; the details are left to the reader. We prove (2.2).

From Lemma 3.2, we see that

$$
\begin{aligned}
d_{0} \bar{\phi}_{n}(x)=\sum_{k=0}^{n-2} \sum_{i=1}^{(n-2) !}(-1)^{\epsilon(i, n-1)+k+1} D_{0, i}^{n-1} d_{k} \sigma(x) \cdot D_{1, i}^{n-1} d_{k-1} \sigma\left(d_{0} x\right) \\
\cdots D_{k, i}^{n-1} d_{0} \sigma\left(d_{0}^{k} x\right) \cdot D_{k, i}^{n-1} \sigma\left(d_{0}^{k+1} x\right) \cdots D_{n-2, i}^{n-1} \sigma\left(d_{0}^{n-1} x\right) .
\end{aligned}
$$

Using (1.1) along with the basic identities defining a simplicial set, we have 


$$
\begin{aligned}
d_{k-j} \sigma\left(d_{0}^{j} x\right) & =\sigma\left(d_{0}^{j} d_{k+1} x\right), & & 0 \leqq j \leqq k, \\
\sigma\left(d_{0}^{j} x\right) & =\sigma\left(d_{0}^{j-1} d_{k+1} x\right), & & k<j<n,
\end{aligned}
$$

and

$$
\begin{aligned}
D_{k, i}^{n-1} d_{0} \sigma\left(d_{0}^{k} x\right) \cdot D_{k, i}^{n-1} \sigma\left(d_{0}^{k+1} x\right) & =D_{k, i}^{n-1}\left[d_{0} \sigma\left(d_{0}^{k} x\right) \cdot \sigma\left(d_{0} d_{0}^{k} x\right)\right] \\
D_{k, i}^{n-1} \sigma\left(d_{1} d_{0}^{k} x\right) & =D_{k, i}^{n-1} \sigma\left(d_{0}^{k} d_{k+1} x\right)
\end{aligned}
$$

so $d_{0} \Phi_{n}(x)$ can be rewritten

$$
\begin{aligned}
\sum_{k=0}^{n-2}(-1)^{k+1} \sum_{i=1}^{(n-2) !}(-1)^{\epsilon(i, n-1)} D_{0, i}^{n-1} \sigma\left(d_{k+1} x\right) \cdot D_{1, i}^{n-1} \sigma\left(d_{0} d_{k+1} x\right) & \cdots D_{n-2, i}^{n-1} \sigma\left(d_{0}^{n-2} d_{k+1} x\right) \\
& =\sum_{j=1}^{n-1}(-1)^{i} \bar{\phi}_{n-1}\left(d_{j} x\right) .
\end{aligned}
$$

4. Proof of Theorem 2.3. Since $(1 \times \mu)$ and $\nabla$ are chain maps, $\partial \psi(x \otimes y)$ can be written as the sum of three terms,

$$
(1 \times \mu) \nabla \sum_{i=1}^{p \prime}(-1)^{\gamma} d_{0} D_{0, i}^{p+1} x \times \tau\left(D_{0, i}^{p+1} x\right) \cdot d_{0} D_{1, i}^{p+1} \sigma(x) \cdots d_{0} D_{p, i}^{p+1} \sigma\left(d_{0}^{p-1} x\right) \otimes y
$$

$$
\begin{aligned}
& +(1 \times \mu) \nabla \sum_{i=1}^{p !} \sum_{j=1}^{p}(-1)^{\gamma+j} d_{j} D_{0, i}^{p+1} x \times d_{j} D_{1, i}^{p+1} \sigma(x) \cdots d_{j} D_{p, i}^{p+1} \sigma\left(d_{0}^{p-1} x\right) \otimes y \\
& +(-1)^{p} \psi(x \otimes \partial y)
\end{aligned}
$$

where $\gamma=\epsilon(i, p+1)+p$.

Consider, for fixed $i$, the expression being summed in the first term of (4.1). That is

$$
d_{0} D_{0, i}^{p+1} x \times \tau\left(D_{0, i}^{p+1} x\right) \cdot d_{0} D_{1, i}^{p+1} \sigma(x) \cdots d_{0} D_{p, i}^{p+1} \sigma\left(d_{0}^{p-1} x\right) \otimes y .
$$

Write $i=m+k(p-1)$ !, $1 \leqq m \leqq(p-1) !, 0 \leqq k \leqq p-1$. It follows easily from (3.1) and Lemma 3.1 that $D_{o, m+k(p-1) !}^{p+1}$ contains an $s_{0}$ unless $k=0$ when $D_{o, m}^{p+1}=D_{o, m}^{p^{\prime}}$. Applying Lemma 3.2 with $k=0$, (4.2) becomes

(4.3) $\quad D_{0, m}^{p} d_{0} x \times \tau\left(D_{0, m}^{p \prime} x\right) \cdot D_{0, m}^{p} \sigma(x) \cdot D_{1, m}^{p} \sigma\left(d_{0} x\right) \cdots D_{p-1, m}^{p} \sigma\left(d_{0}^{p-1} x\right) \otimes y$.

By Lemma 3.1 and the last part of Lemma 1.2, we see that $\tau\left(D_{o, m}^{p^{\prime}} x\right) \cdot D_{0, m}^{p} \sigma(x)$ $=D_{o, m}^{p} \tau(x) \cdot D_{o, m}^{p} \sigma(x)=1$, so $(4.3)$ becomes

$$
D_{0, m}^{p} d_{0} x \times D_{1, m}^{p} \sigma\left(d_{0} x\right) \cdots D_{p-1, m}^{p} \sigma\left(d_{0}^{p-2} d_{0} x\right) \otimes y .
$$


If $k>0, \tau\left(D_{o, m+k(p-1) ! x}^{p+1}:=1\right.$, so by Lemma 3.2, (4.2) can be rewritten as

$$
\begin{aligned}
& D_{0, m}^{p} d_{k} x \times D_{1, m}^{p} d_{k-1} \sigma(x) \cdots D_{k, m}^{p} d_{0} \sigma\left(d_{0}^{k-1} x\right) \\
& \quad \cdot D_{k, m}^{p} \sigma\left(d_{0}^{k} x\right) \cdots D_{p-1, m}^{p} \sigma\left(d_{0}^{p-1} x\right) \otimes y .
\end{aligned}
$$

Using (1.1), we have

$$
\begin{aligned}
D_{k, m}^{p} d_{0} \sigma\left(d_{0}^{k-1} x\right) \cdot D_{k, m}^{p} \sigma\left(d_{0}^{k} x\right) & =D_{k, m}^{p} \sigma\left(d_{1} d_{0}^{k-1} x\right) \\
& =D_{k, m}^{p} \sigma\left(d_{0}^{k-1} d_{k} x\right) .
\end{aligned}
$$

Thus, (4.4) becomes

$$
D_{0, m}^{p} d_{k} x \times D_{1, m}^{p} \sigma\left(d_{k} x\right) \cdots D_{p-1, m}^{p} \sigma\left(d_{0}^{p-2} d_{k} x\right) \otimes y
$$

and the first term of (4.1) can be rewritten

$(1 \times \mu) \nabla \sum_{m=1}^{(p-1) !} \sum_{k=0}^{p-1}(-1)^{\epsilon(i, p+1)+p} D_{0, m}^{p} d_{k} x \times D_{1, m}^{p} \sigma\left(d_{k} x\right) \cdots D_{p-1, m}^{p} \sigma\left(d_{0}^{p-2} d_{k} x\right) \otimes y$ which, by (3.2), is easily seen to be $\psi(\partial x \otimes y)+(-1)^{p} \psi\left(d_{p} x \otimes y\right)$.

We now turn our attention to the second term of (4.1). By Lemma 3.4, this term reduces to

$$
(1 \times \mu) \nabla \sum(-1)^{\epsilon(i, p+1)} d_{p} D_{0, i}^{p+1} x \times d_{p} D_{1, i}^{p+1} \sigma(x) \cdots D_{p, i}^{p+1} \sigma\left(d_{0}^{p-1} x\right) \otimes y
$$

which, using Lemma 3.3 , is equal to

$$
(1 \times \mu) \nabla(1 \times \pi \otimes 1)(\nabla \otimes 1) \sum_{r=1}^{p} \sum_{s=1}^{(r-1) !}(-1)^{\gamma} D_{0.8}^{r} d_{r}^{p+1-r} x
$$

$$
\times D_{1, s}^{r} \sigma\left(d_{r}^{p+1-r} x\right) \cdots D_{r-1,8}^{r} \sigma\left(d_{0}^{r-2} d_{r}^{p+1-r} x\right) \otimes \bar{\phi}_{p+1-r}\left(d_{0}^{r-1} x\right) \otimes y,
$$

where $\gamma=\epsilon(s, r)+p+r+1$. Now we need the following lemma.

Lemma 4.1. The following diagram is commutative.

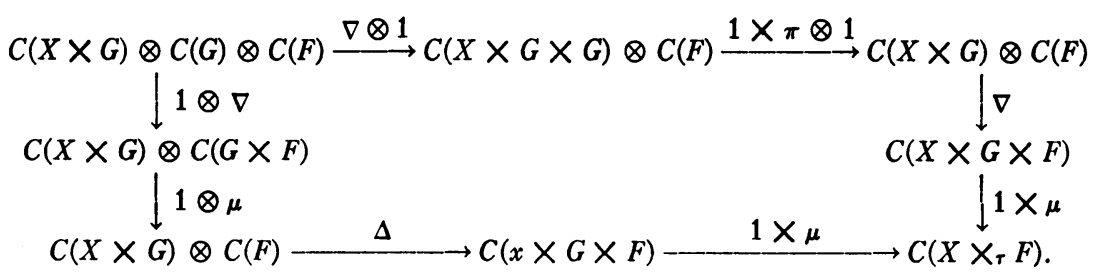

Proof. Insert $C(X \times G \times G \times F)$ into the center of the diagram connected 
to the midpoints of the four sides by the obvious maps. The four resulting diagrams are easily seen to be commutative which proves the lemma.

Now, using Lemma 4.1, (4.5) can be rewritten

$$
\begin{aligned}
(1 \times \mu) \nabla \sum_{r=1}^{p} & \sum_{8=1}^{(r-1) !}(-1)^{\gamma} D_{0,8}^{r} d_{r}^{p+1-r} x \times D_{1,8}^{r} \sigma\left(d_{r}^{p+1-r} x\right) \cdots D_{r-1,8}^{r} \sigma\left(d_{0}^{r-2} d_{r}^{p+1-r} x\right) \\
& \otimes \mu \nabla\left[\Phi_{p+1-r}\left(d_{0}^{r-1} x\right) \otimes y\right] \\
& =(-1)^{p} \sum_{r=1}^{p} \psi\left(d_{r}^{p+1-r} x \otimes \mu \nabla\left[\bar{\phi}_{p+1-r}\left(d_{0}^{r+1} x\right) \otimes y\right]\right) \\
& =(-1)^{p} \psi(1 \otimes \mu \nabla)(1 \otimes \phi \otimes 1)(\Delta \otimes 1)(x \otimes y)+(-1)^{p} \psi\left(d_{p} x \otimes y\right) \\
& =(-1)^{p} \psi(x \otimes y \cap \phi)+(-1)^{p} \psi\left(d_{p} x \otimes y\right) .
\end{aligned}
$$

Thus, $\partial \psi(x \otimes y)=\psi\left(\partial_{\phi}(x \otimes y)\right)$ and the theorem is proved.

5. Proof of Theorem 2.4. Define filtrations

$$
\begin{array}{rlrl}
A_{p} & =\sum_{q \leqq p} C_{q}(X)_{\phi} \otimes C(F), & & p \geqq 0, A_{-1}=0, \\
B_{p}=C\left(X^{p} \times_{\tau} F\right), & p \geqq 0, B_{-1}=0,
\end{array}
$$

where $X^{p}$ is the simplicial subset of $X$ generated by all $q$ simplicies, $q \leqq p$. As is easily verified, $\partial_{\phi} A_{p} \subset A_{p}, \partial B_{p} \subset B_{p}$, and $\psi A_{p} \subset B_{p}$. Let $\psi^{\prime}: A_{p} / A_{p-1}$ $\rightarrow B_{p} / B_{p-1}$ be the map induced by $\psi$. The following lemma is not difficult $[4$, p. 106].

LEMмA 5.1. If $\psi_{*}^{\prime}: H\left(A_{p} / A_{p-1}\right) \rightarrow H\left(B_{p} / B_{p-1}\right)$ is an isomorphism for all $p \geqq 0$, then $\psi_{*}: H\left(A_{p}\right) \rightarrow H\left(B_{p}\right)$ is an isomorphism for all $p \geqq 0$.

Since $H_{r}\left(C(X)_{\phi} \otimes C(F)\right) \approx H_{r}\left(A_{p}\right), p>r$ and $H_{r}\left(C\left(X \times_{\tau} F\right)\right) \approx H_{r}\left(B_{p}\right)$, $p>r$, we have

CoRollary 5.2. If $\psi_{*}^{\prime}: H_{*}\left(A_{p} / A_{p-1}\right) \rightarrow H_{*}\left(B_{p} / B_{p-1}\right)$ is an isomorphism for all $p \geqq 0$, then $\psi_{*}: H_{*}\left(C(X)_{\phi} \otimes C(F)\right) \rightarrow H_{*}\left(C\left(X X_{\tau} F\right)\right)$ is an isomorphism.

Now, consider the maps $f, \nabla$, and $H$ of Eilenberg and MacLane as maps

$$
\begin{aligned}
f: C\left(X \times_{\tau} F\right) & \rightarrow C(X)_{\phi} \otimes C(F), \\
\nabla: C(X)_{\phi} \otimes C(F) & \rightarrow C\left(X \times_{\tau} F\right), \\
H: C\left(X \times_{\tau} F\right) & \rightarrow C\left(X \times_{\tau} F\right) .
\end{aligned}
$$

In general, $f$ and $\nabla$ will no longer be chain maps and $H$ will not be a chain homotopy between $\nabla f$ and the identity. However, it is easily verified that these maps do preserve the filtrations defined above so induce maps $f^{\prime}, \nabla^{\prime}$, and $H^{\prime}$ on the quotients $A_{p} / A_{p-1}$ and $B_{p} / B_{p-1}$.

Lемма 5.3. The maps $f^{\prime}$ and $\nabla^{\prime}$ are chain maps with $f^{\prime} \nabla^{\prime}=$ identity and $H^{\prime}$ is a chain homotopy with $\partial H^{\prime}+H^{\prime} \partial=\nabla^{\prime} f^{\prime}-i d e n t i t y$. 
Proof. We notice that, for $x \otimes y \in A_{p}, x \times y \in B_{p}$,

$$
\partial \phi(x \otimes y)=(-1)^{p} x \otimes \partial y \bmod A_{p-1}
$$

and

$$
\partial(x+y)=\sum_{i=0}^{p}(-1)^{i} d_{i} x \times d_{i} y \bmod B_{p-1} .
$$

The first of these equations follows from the fact that $\partial x \otimes y$ and $x \otimes y \cap \phi$ are in $A_{p-1}$; the second from the fact that $d_{0}(x \times y)=d_{0} x \times d_{0} y \bmod B_{p-1}$.

Thus, the differentials on $A_{p} / A_{p-1}$ and $B_{p} / B_{p-1}$ are essentially the standard nontwisted differentials so the properties of $f^{\prime}, \nabla^{\prime}$, and $H^{\prime}$ listed in the lemma follow from the corresponding properties of $f, \nabla$, and $H$.

We now show $\psi^{\prime}$ has a very simple form not very different from $\nabla^{\prime}$.

Lemma 5.4. For any $n>0, D_{0,1}^{n}=i d e n t i t y, D_{j, 1}^{n}=s_{0}^{j}$ for $0<j \leqq p, D_{o, i}^{n}$ contains a face operator for $i>1$, and $\epsilon(1, n)=n-1 \bmod 2$.

This lemma follows by a simple induction from (3.1).

Corollary 5.5. The map $\psi^{\prime}$ is given by

$$
\psi^{\prime}(x \otimes y)=\sum(-1)^{\operatorname{sgn}(\mu, \nu)} s_{\mu} x \times s_{\mu}\left[s_{0} \sigma(x) \cdots s_{0}^{r} \sigma\left(d_{0}^{r-1} x\right)\right] \cdot s_{\nu} y
$$

where $x \in C_{p}(X), y \in C_{q}(F)$, and the sum is over all $(q, p)$ shuffles $(\mu, \nu)$.

Proof. By definition,

$$
\psi^{\prime}(x \otimes y)=\sum(-1)^{\gamma} s_{\mu} D_{0, i}^{p+1} x \times s_{\mu}\left[D_{1, i}^{p+1} \sigma(x) \cdots D_{p, i}^{p+1} \sigma\left(d_{0}^{p-1} x\right)\right] \cdot s_{\nu} y,
$$

where the sum is over $i=1$ to $p$ ! and all $(q, p)$ shuffles $(\mu, \nu)$ and $\gamma=\operatorname{sgn}(\mu, \nu)$ $+\epsilon(i, p+1)+p$. Since $D_{o, i}^{p+1}$ contains a face operator for $i>1$, all terms in the sum with $i>1$ will be in $B_{p-1}$. The form of the remaining terms follows directly from Lemma 5.4 .

Let $g: B_{p} / B_{p-1} \rightarrow B_{p} / B_{p-1}$ be defined by

$$
g\left(s_{\mu} x \times y\right)=s_{\mu} x \times s_{\mu}\left[s_{0} \sigma(x) \cdots s_{0}^{p} \sigma\left(d_{0}^{p-1} x\right)\right] \cdot y
$$

where $s_{\mu}=s_{i_{r}} \cdots s_{i_{1}}, i_{r}>\cdots>i_{1}, x$ is a nondegenerate element of $X_{p}$ and $y \in F_{p+r}$. It is easily seen that $g$ commutes with the face operators and thus with $\partial$. In fact, $g$ is a chain isomorphism with inverse $h: B_{p} / B_{p-1} \rightarrow B_{p} / B_{p-1}$ defined by

$$
h\left(s_{\mu} x \times y\right)=s_{\mu} x \times s_{\mu}\left[s_{0}^{r} \tau\left(d_{0}^{r-1} x\right) \cdots s_{0} \tau(x)\right] \cdot y
$$

where $x, y$, and $s_{\mu}$ are as above.

Now, we observe that $g \nabla^{\prime}=\psi^{\prime}$ and thus $g_{*} \nabla_{*}^{\prime}=\psi_{*}^{\prime}$. By Lemma 5.3 and 
the fact that $g$ is an isomorphism, we see that $\nabla_{*}^{\prime}, g_{*}$ and therefore $\psi_{*}^{\prime}$ are isomorphisms. Applying Corollary 5.2 completes the proof of Theorem 2.4.

\section{PART II}

6. Fiber bundles over spheres. It is well known that equivalence classes of fiber bundles over the $n$-sphere $S^{n}$ with a connected group $G$ are in a 1-1 correspondence with the elements of $\Pi_{n-1}(G)\left[10\right.$, p. 99]. If $p: E \rightarrow S^{n}$ is a fiber bundle with group $G$ and fiber $F$, the corresponding element of $\Pi_{n-1}(G)$ can be obtained in the following manner.

Let $p^{\prime}: E^{\prime} \rightarrow S^{n}$ be the principle $G$-bundle associated with $p: E \rightarrow S^{n}$. Let $x_{0}$ be a point of $S^{n}$ and identify $p^{\prime-1}\left(x_{0}\right)$ with $G$. Passing to singular complexes, we obtain a simplicial fiber map $S\left(p^{\prime}\right): S\left(E^{\prime}\right) \rightarrow S\left(S^{n}\right)$. Suppose $T:\left(\Delta^{n}, \partial \Delta^{n}\right)$ $\rightarrow\left(S^{n}, x_{0}\right)$ is a characteristic map for $S^{n}$ (i.e. $T \mid$ Int. $\Delta^{n}$ is a homomorphism) and $\tilde{T}$ in $S_{n}\left(E^{\prime}\right)$ by requiring that $S\left(p^{\prime}\right) \tilde{T}=T$ and $d_{i} \tilde{T}=s_{0}^{n-1} e, 0<i \leqq n, e$ the 0 -simplex at the identity of $G$. Then $d_{0} T=\alpha:\left(\Delta^{n-1}, \partial \Delta^{n-1}\right) \rightarrow(G, e)$ represents the element of $\Pi_{n-1}(G)$ associated with $p: E \rightarrow S^{n}$.

Let $\bar{\Delta}^{n}$ be the simplicial subset of $\Delta^{n}$ generated by all simplicies $x \in \Delta_{q}^{n}$, $q<n$. (In this paragraph, $\Delta^{n}$ denotes the simplicial set representing the standard $n$-simplex.) Define $\Sigma^{n}=\Delta^{n} / \bar{\Delta}^{n}$. $\Sigma^{n}$ has only two nondegenerate simplicies, $b$ in $\Sigma_{0}^{n}$ and $\sigma_{n}$ in $\Sigma_{n}^{n}$. Now, considering $\alpha$ as an element of $S_{n-1}(G)$, define a mapping $\tau: \Sigma^{n} \rightarrow S(G)$ by

$$
\begin{array}{rlr}
\tau\left(s_{0}^{q} b\right) & =s_{0}^{q-1} e, \\
\tau\left(\sigma_{n}\right) & =\alpha, \\
\tau\left(s_{i_{r}} \cdots s_{i_{1}} \sigma_{n}\right) & = \begin{cases}s_{i_{r}-1} \cdots s_{i_{1}-1} \alpha, \\
s_{0}^{n+r-1} e,\end{cases} & i_{1}>0
\end{array}
$$

where $i_{r}>\cdots>i_{1}$. It is easily verified that $\tau$ is a twisting function. Since $G$ acts on $F, S(G)$ acts on $S(F)$ and we can define $\Sigma^{n} \times r S(F)$.

TheOREM 6.1. There exist simplicial maps $g: \Sigma^{n} \rightarrow S\left(S^{n}\right), f: \Sigma^{n} X_{\tau} S(F)$ $\rightarrow S(E)$ inducing isomorphisms on homology and giving a commutative diagram

$$
\begin{aligned}
& \Sigma^{n} \times_{\tau} S(F) \stackrel{f}{\rightarrow} S(E) \\
& \pi \downarrow \quad g \quad \downarrow S(p) \\
& \Sigma^{n} \stackrel{g}{\longrightarrow} S\left(S^{n}\right)
\end{aligned}
$$

where $\pi(x, y)=x$.

The following two lemmas will be needed in the proof of Theorem 6.1. Proofs can be found in $[9$, p. 39]. 
Lemma 6.2. Let $p: E \rightarrow B$ be a principal G-bundle. Then there is an action $\mu: E \times G \rightarrow E$ mapping fibers into fibers with the property that, restricted to $a$ fiber, the action reduces to multiplication on the right.

Leмma 6.3. Let $p: E \rightarrow B$ be a principal $G$ bundle and suppose $G$ acts effectively on $F$. Define an equivalence relation $\sim$ in $E \times F$ by $(x g, y) \sim(x, g y)$ and define $\bar{p}: E \times F / \sim=\bar{E} \rightarrow B$ by $\bar{p}(x, y)=p(x)$. Then $\bar{p}: \bar{E} \rightarrow B$ is the bundle with fiber $F$ and group $G$ associated with $p: E \rightarrow B$.

Proof of Theorem 6.1. Suppose $p: E \rightarrow S^{n}$ is a principal bundle and $p: \bar{E}=E \times F / \sim \rightarrow S^{n}$ the associated bundle with fiber $F$. Let $\lambda: E \times F \rightarrow \bar{E}$ be the natural map and define $g: \Sigma^{n} \rightarrow S\left(S^{n}\right)$ and $f: \Sigma^{n} \times{ }_{\tau} S(F) \rightarrow S(E)$ by

$$
\begin{aligned}
g\left(s_{0}^{q} b\right) & =\text { the singular } q \text {-simplex at } x_{0}, \\
g\left(s_{i_{r}} \cdots s_{i_{1}} \sigma_{n}\right) & =s_{i_{r}} \cdots s_{i_{1}} T, \\
f\left(s_{0}^{q} b \times y\right) & =S(\lambda)\left(s_{0}^{q} e \times y\right), \\
f\left(s_{i_{r}} \cdots s_{i_{1}} \sigma_{n} \times y\right) & =S(\lambda)\left(s_{i_{r}} \cdots s_{i_{1}} \tilde{T} \times y\right),
\end{aligned}
$$

where $i_{r}>\cdots>i_{1}$ and $y \in S(F)$. The verifications that $f$ and $g$ are simplicial maps are straightforward with the exception $d_{0} f=f d_{0}$. In this case we have

$$
d_{0} f\left(s_{i_{r}} \cdots s_{i_{1}} \sigma_{n} \times y\right)= \begin{cases}S(\lambda)\left(s_{i_{r}-1} \cdots s_{i_{2}-1} \tilde{T} \times d_{0} y\right), & i_{1}=0 \\ S(\lambda)\left(s_{i_{r}-1} \cdots s_{i_{1}-1} \alpha \times d_{0} y\right), & i_{1}>0\end{cases}
$$

and

$$
f d_{0}\left(s_{i_{r}} \cdots s_{i_{1}} \sigma_{n} \times y\right)= \begin{cases}S(\lambda)\left(s_{i_{r}-1} \cdots s_{i_{2}-1} T \times d_{0} y\right), & i_{1}=0 \\ S(\lambda)\left(s_{0}^{n+r-1} e \times s_{i_{r}-1} \cdots s_{i_{1}-1} \alpha \cdot d_{0} y\right), & i_{1}>0 .\end{cases}
$$

These terms are equal since $S(\lambda)(x g, y)=S(\lambda)(x, g y)$.

The commutativity of (6.1) is trivial as is the fact that $g$ induces isomorphisms on homology. To prove $f$ induces an isomorphism on homology, we note that $f \mid S(F)$ is the identity and that $f$ and $g$ induce maps on the standard spectral sequences of $\pi: \Sigma^{n} \times{ }_{r} S(F) \rightarrow \Sigma^{n}$ and $S(p): S(E) \rightarrow S\left(S^{n}\right)$. Thus, by the comparison theorem, since $g$ and $f \mid S(F)$ induce isomorphisms on homology, so does $f$.

To prove the theorem for principal bundles, we define $g$ as above and $f$ by replacing $S(\lambda)$ by $S(\mu), \mu: E \times G \rightarrow E$. The remainder of the proof is exactly as above.

7. The Wang sequences. Applying Theorems 2.4 and 6.1 , we know that the composite

$$
H_{*}\left(C\left(\Sigma^{n}\right)_{\phi} \otimes C(F)\right) \stackrel{\psi_{*}}{\rightarrow} H_{*}\left(\Sigma^{n} \times_{\tau} S(F)\right) \stackrel{f_{*}}{\rightarrow} H_{*}(E)
$$


is an isomorphism. The following two lemmas tell us that the twisting cochain of $C\left(\Sigma^{n}\right)_{\phi} \otimes C(F)$ and the map $\psi: C\left(\Sigma^{n}\right)_{\phi} \otimes C(F) \rightarrow C\left(\Sigma^{n} X_{\top} S(F)\right)$ have a very simple form.

LemMa 7.1. The twisting cochain $\phi$ of $C\left(\Sigma^{n}\right)_{\phi} \otimes C(F)$ has the form

$$
\begin{array}{rlrl}
\phi_{n}\left(\sigma_{n}\right) & =(-1)^{n+1} \alpha^{-1}, \\
\phi_{q} & =0, & q \neq n .
\end{array}
$$

Lemma 7.2. The map $\psi: C\left(\Sigma^{n}\right)_{\phi} \otimes C(F) \rightarrow C\left(\Sigma^{n} X_{\tau} S(F)\right)$ has the form

$$
\begin{aligned}
\psi(b \otimes c) & =s_{0}^{p} b \times c, \\
\psi\left(\sigma_{n} \otimes c\right) & =\sum(-1)^{\operatorname{sgn}(\mu, \nu)} s_{\mu} \sigma_{n} \times s_{\mu} s_{0} \alpha^{-1} \cdot s_{\nu} c,
\end{aligned}
$$

where $c \in C_{p}(F)$ and the sum is over all $(p, n)$ shuffles $(\mu, \nu)$.

The proofs of Lemmas 7.1 and 7.2 are almost identical with the proof of Corollary 5.5 , so we omit them here.

Now, using Lemma 7.1 we see that

$$
\begin{aligned}
\partial_{\phi}(b \otimes c) & =b \otimes \partial c \\
\partial_{\phi}\left(\sigma_{n} \otimes c\right) & =(-1)^{n} \sigma_{n} \otimes \partial c-b \otimes \alpha^{-1} * c
\end{aligned}
$$

where

$$
\alpha^{-1} * c=\mu \nabla\left(\alpha^{-1} \otimes c\right), \quad \mu: G \times F \rightarrow F .
$$

Define maps $\lambda: C(F) \rightarrow C\left(\Sigma^{n}\right)_{\phi} \otimes C(F), \eta: C\left(\Sigma^{n}\right)_{\phi} \otimes C(F) \rightarrow C(F)$ by $\lambda(x)$ $=b \otimes x, \eta\left(b \otimes x+\sigma_{n} \otimes y\right)=(-1)^{n} y$. As is easily seen, $\lambda$ is a chain monomorphism of degree $0, \eta$ is a chain epimorphism of degree $-n$, and kernel $\eta$ $=$ image $\lambda$. Therefore, we have an exact sequence

$$
0 \rightarrow C(F) \rightarrow C\left(\Sigma^{n}\right)_{\phi} \otimes C(F) \rightarrow C(F) \rightarrow 0 .
$$

Passing to homology, we have an exact triangle

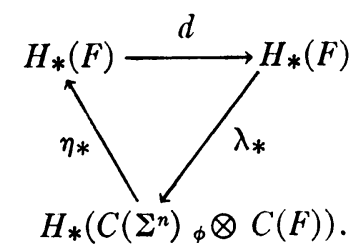

Identifying $H_{*}\left(C\left(\Sigma^{n}\right)_{\phi} \otimes C(F)\right)$ with $H_{*}(E)$ under the map $f_{*} \psi_{*}$, we obtain the homology Wang sequence

$$
\cdots \rightarrow H_{p}(F) \stackrel{\gamma}{\rightarrow} H_{p+n-1}(F) \stackrel{j_{*}}{\rightarrow} H_{p+n-1}(E) \stackrel{\zeta}{\rightarrow} H_{p-1}(E) \rightarrow \cdots
$$

where $j_{*}$ is induced by $j: F \subset E, \zeta(c)=\eta_{*} \psi_{*}^{-1} f_{*}^{-1}(c)$, and $\gamma(c)=(-1)^{n} \alpha * c$, 
$\alpha \in H_{n-1}(G)$ is the homology characteristic class of $p: E \rightarrow S^{n}$.

Before investigating $H^{*}(E)$, we define a map dual to the product $H_{*}(G) \otimes H_{*}(F) \rightarrow H_{*}(F)$. Consider $a$ in $C_{q}(G), u \in C^{p}(F)$ and define $a \wedge u$ in $C^{p-q}(F)$ by the formula $(a \wedge u)(c)=u(a * c), c$ in $C_{p-q}(F)$. As is easily verified, $\delta(a \wedge u)=(-1)^{q}(a \wedge \delta u-\partial a \wedge u)$. Therefore, if $a$ is a cycle and $u$ a cocycle, $a \wedge u$ is a cocycle and modifying $a$ by a boundary and $u$ by a coboundary clearly changes $a \wedge u$ by a coboundary. Thus we have a map $H_{q}(G) \otimes H^{p}(F)$ $\rightarrow H^{p-q}(F)$.

Now, consider $C^{*}(E)$. By Theorems 2.4 and 6.1 , and the fact that $C\left(\Sigma^{n}\right)$ is finitely generated, $C^{*}(E)$ is chain equivalent to $C^{*}\left(\Sigma^{n}\right)_{\phi} \otimes C^{*}(F)$. Let $b^{*}$ in $C^{0}\left(\Sigma^{n}\right), \sigma^{n}$ in $C^{n}\left(\Sigma^{n}\right)$ be generators with $b^{*}(b)=\sigma^{n}\left(\sigma_{n}\right)=1$. Then, from 7.1, we have

$$
\begin{aligned}
& \delta_{\phi}\left(b^{*} \otimes u\right)=b^{*} \otimes \delta u-\sigma^{n} \otimes \alpha^{-1} * u, \\
& \delta_{\phi}\left(\sigma^{n} \otimes u\right)=(-1)^{n} \sigma^{n} \otimes \delta u .
\end{aligned}
$$

Define maps $\bar{\lambda}: C^{*}\left(\Sigma^{n}\right)_{\phi} \otimes C^{*}(F) \rightarrow C^{*}(F)$, and $\bar{\eta}: C^{*}(F) \rightarrow C^{*}\left(\Sigma^{n}\right)_{\phi} \otimes C^{*}(F)$ by $\bar{\lambda}\left(b \otimes u+\sigma^{n} \otimes v\right)=u$ and $\bar{\eta}(u)=(-1)^{n} \sigma^{n} \otimes u$. Now, it is easy to see that the sequence

$$
0 \rightarrow C^{*}(F) \rightarrow C^{*}\left(\Sigma^{n}\right)_{\phi} \otimes C^{*}(F) \rightarrow C^{*}(F) \rightarrow 0
$$

is exact so, just as above, we obtain the cohomology Wang sequence

$$
\ldots \rightarrow H^{p}(F) \stackrel{\gamma^{*}}{\rightarrow} H^{p-n+1}(F) \stackrel{\zeta^{*}}{\rightarrow} H^{p+1}(E) \stackrel{j^{*}}{\rightarrow} H^{p+1}(F) \rightarrow \cdots
$$

where $j^{*}$ is induced by $j: F \subset E, \gamma^{*}(u)=(-1)^{n} \alpha \bigwedge u$, and $\zeta^{*}(u)=f^{*-1} \psi^{*-1} \bar{\eta}^{*}(u)$, $\alpha$ the homology characteristic class.

8. Products in the cohomology Wang sequence. As a module, $C\left(\Sigma^{n}\right)_{\phi}$ $\otimes C(F)$ is the ordinary tensor product of $C\left(\Sigma^{n}\right)$ with $C(F)$, both of which are D.G.A. coalgebras. Therefore, we can define a module map

$$
\Delta: C\left(\Sigma^{n}\right)_{\phi} \otimes C(F) \rightarrow C\left(\Sigma^{n}\right)_{\phi} \otimes C(F) \otimes C\left(\Sigma^{n}\right)_{\phi} \otimes C(F)
$$

by $\Delta=(1 \otimes T \otimes 1)\left(\Delta_{1} \otimes \Delta_{2}\right)$ where $\Delta_{1}$ and $\Delta_{2}$ are the coproducts in $C\left(\Sigma^{n}\right)$ and $C(F)$ respectively (given by $c \rightarrow f(c \times c), f$ the Eilenberg and MacLane map) and $T(x \otimes y)=(-1)^{p q} y \otimes x, \operatorname{dim} x=p$, $\operatorname{dim} y=q$.

Lemma 8.1. $C\left(\Sigma^{n}\right)_{\phi} \otimes C(F)$ is a D.G.A. coalgebra with coproduct $\Delta$ defined above.

Proof. First observe that, for $c \in C_{p}(F)$,

$\Delta\left(b \otimes \alpha^{-1} * c\right)$

$$
\left.=\sum_{i=0}^{p}\left[b \otimes \alpha^{-1} * d_{i+1}^{p-i} c \otimes b \otimes d_{0}^{i} c+(-1)^{(n-1) i} b \otimes d_{i+1}^{p-i} c \otimes b \otimes \alpha^{-1} * d_{0}^{i} c\right)\right]
$$


Now,

$\left(\partial_{\phi} \otimes 1+\omega \otimes \partial_{\phi}\right) \Delta\left(b \otimes x+\sigma_{n} \otimes y\right)=(\bar{\partial} \otimes 1+\omega \otimes \bar{\partial}) \Delta\left(b \otimes x+\sigma_{n} \otimes y\right)$ $+\sum_{i=0}^{p}\left[b \otimes \alpha^{-1} * d_{i+1}^{p-i} y \otimes b \otimes d_{0}^{i} y+(-1)^{n i+i} b \otimes d_{i+1}^{p-i} y \otimes b \otimes \alpha^{-1} * d_{0}^{i} y\right]$ where $\bar{\partial}$ is the usual tensor product differential, and $\omega(x)=(-1)^{p} x, \operatorname{dim} x=p$. As is well known, $(\bar{\partial} \otimes 1+\omega \otimes \bar{\partial}) \Delta=\Delta \bar{\partial}$ so

$$
\begin{aligned}
\left(\partial_{\phi} \otimes 1+\omega \otimes \partial_{\phi}\right) \Delta\left(b \otimes x+\sigma_{n} \otimes y\right) & =\Delta \bar{\partial}\left(b \otimes x+\sigma_{n} \otimes y\right)-\Delta\left(b \otimes \alpha^{-1} * y\right) \\
& =\Delta \partial_{\phi}\left(b \otimes x+\sigma_{n} \otimes y\right) .
\end{aligned}
$$

THEOREM 8.2. The map $f_{*} \psi_{*}: C\left(\Sigma^{n}\right)_{\phi} \otimes C(F) \rightarrow C(E)$ is a coalgebra map.

Corollary 8.3. The map $\psi^{*} f^{*}: C^{*}(E) \rightarrow C^{*}\left(\Sigma^{n}\right)_{\phi} \otimes C^{*}(F)$ is an algebra map.

The proof of Theorem 8.2 will be given in the next section. The corollary follows immediately from the definition of cup product.

We now investigate the behavior of the maps in the cohomology Wang sequence with respect to cup products. The behavior of $j^{*}$ is of course very nice; it is a ring homomorphism. Almost as well behaved is the map $\gamma^{*}$ (see [6, p. 209]).

Theorem 8.4. Consider $u$ in $H^{p}(F)$ and $v$ in $H^{q}(F)$. Then

$$
\gamma^{*}(u \cup v)=\gamma^{*}(u) \cup v+(-1)^{(n-1) p} u \cup \gamma^{*}(v) .
$$

Proof. Let $\bar{u}$ and $\bar{v}$ be cocycles representing $u$ and $v$ respectively. Then, for $c$ in $C_{r}(F)$,

$$
\begin{aligned}
& \left.\left(\gamma^{*}\right)(\bar{u} \cup \bar{v})\right)(c)=(-1)^{n}(\bar{u} \cup \bar{v})(\alpha * c)=(-1)^{n} \mu(\bar{u} \otimes \bar{v}) \Delta(\alpha * c) \\
& =(-1)^{n} \sum_{i=0}^{r} \mu(\bar{u} \otimes \bar{v})\left(\alpha * d_{i+1}^{r-i} c \otimes d_{0}^{i} c+(-1)^{(n-1) i} d_{i+1}^{r-i} c \otimes \alpha * d_{0}^{i} c\right) \\
& =(-1)^{n} \sum_{i=0}^{r} \mu\left(\bar{u}\left(\alpha * d_{i+1}^{r-i} c\right) \otimes \bar{v}\left(d_{0}^{i} c\right)+(-1)^{(n-1) i} \bar{u}\left(d_{i+1}^{r-i} c\right) \otimes \bar{v}\left(\alpha * d_{0}^{i} c\right)\right)
\end{aligned}
$$

Since $u\left(d_{i+1}^{r-i} c\right)=0$ for $i \neq p$ and $v\left(d_{0}^{i} c\right)=0$ for $i \neq r-q$, this becomes

$$
\gamma^{*}(\bar{u}) \cup \bar{v}+(-1)^{(n-1) q} \bar{u} \cup \gamma^{*}(\bar{v}) \text {. }
$$

We now turn our attention to the $\operatorname{map}\left({ }^{4}\right) \zeta^{*}$.

Theorem 8.5. Consider $u$ in $H^{p}(F)$ and $v$ in $H^{q}(E)$. Then

(4) Theorem 8.5 is an analogue of Massey's Lemma 1 of [8]. 


$$
\begin{aligned}
& \zeta^{*}\left(u \cup j^{*}(v)\right)=\zeta^{*}(u) \cup v, \\
& \zeta^{*}\left(j^{*}(v) \cup u\right)=(-1)^{n q} v \cup \zeta^{*}(u)
\end{aligned}
$$

Proof. Since the second equation follows from the first by the commutativity of cup products, we only prove the first. By Corollary 8.3 and the definition of $\zeta^{*}$, this is equivalent to proving that $\bar{\eta}^{*}\left(u \cup j^{*}(v)\right)=\bar{\eta}^{*}(u) \cup \psi^{*} f^{*}(v)$. Let $u_{1}$ in $C^{*}(F)$ be a cocycle representing $u$ and $b^{*} \otimes v_{1}+\sigma^{n} \otimes v_{2}$ in $C^{*}\left(\Sigma^{n}\right)_{\phi} \otimes C^{*}(F)$ a cocycle representing $\psi^{*} f^{*}(v)$. Now, since $j^{*}=\bar{\lambda}^{*} \psi^{*} f^{*}$, $\bar{\eta}^{*}\left(u \cup j^{*}(v)\right)$ is represented by $\bar{\eta}^{*}\left(u_{1} \cup \bar{\lambda}^{*}\left(b^{*} \otimes v_{1}+\sigma^{n} \otimes v_{2}\right)\right)$ which, by the definitions of $\bar{\eta}^{*}$ and $\bar{\lambda}^{*}$ is $(-1)^{n} \sigma^{n} \otimes u_{1} \cup v_{1}$.

Again by the definition of $\bar{\eta}^{*}, \bar{\eta}^{*}(u) \cup \psi^{*} f^{*}(v)$ is represented by $\bar{\eta}^{*}\left(u_{1}\right)$ $\cup\left(b^{*} \otimes v_{1}+\sigma^{n} \otimes v_{2}\right)=(-1)^{n} \sigma^{n} \otimes u_{1} \cup v_{1}$ which proves the theorem.

Corollary 8.6. $\zeta^{*} H(F)$ is an ideal in which all products are zero.

Proof. That $\zeta^{*} H(F)$ is an ideal follows immediately from Theorem 8.5. To see that all products are zero, consider $u, v$ in $H^{*}(F)$. Then

$$
\zeta^{*}(u) \cup \zeta^{*}(v)=\zeta^{*}\left(u \cup j^{*} \zeta^{*}(v)\right)=0
$$

since $j^{*} \zeta^{*}=0$.

Corollary 8.7. Let $s^{n}$ denote the generator of $H^{n}\left(S^{n}\right)$ and $v$ in $H^{*}(E)$. Then $\zeta^{*} j^{*}(v)=p^{*}\left(s^{n}\right) \cup v$.

Proof. Letting $u=1$ in $H^{0}(F)$, we have

$$
\zeta^{*} j^{*}(v)=\zeta^{*}\left(1 \cup j^{*}(v)\right)=\zeta^{*}(1) \cup v .
$$

It is easily seen that $\zeta^{*}(1)=p^{*}\left(s^{n}\right)$, proving the corollary.

Before closing this section, we note a consequence of Corollary 8.7. Writing the cohomology Wang sequence as an exact triangle,

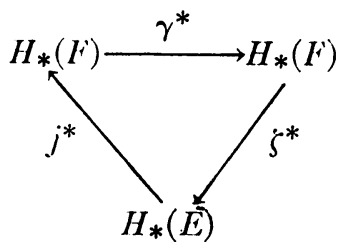

we see it actually defines an exact couple [7]. Thus we have an associated spectral sequence with $E_{1}=H^{*}(E), E_{2}=H\left(H^{*}(E), d\right)$ where $d=\zeta^{*} j^{*}$. By Corollary $8.7, \zeta^{*} j^{*}(u)=p^{*}\left(s^{n}\right) \cup u$ so

$$
E_{2}=A_{n}\left(p^{*}\left(s^{n}\right)\right) /\left\langle p^{*}\left(s^{n}\right)\right\rangle
$$

where $A_{n}\left(p^{*}\left(s^{n}\right)\right)=\left\{u \in H(E)\right.$ with $\left.p^{*}\left(s^{n}\right) \cup u=0\right\}$ and $\left\langle p^{*}\left(s^{n}\right)\right\rangle$ is the ideal generated by $p^{*}\left(s^{n}\right)$.

9. Proof of Theorem 8.2. Since $f$ is a simplicial map $f_{*}$ is clearly a coalgebra map. Thus we need only show that the diagram 


$$
\begin{gathered}
C\left(\Sigma^{n}\right)_{\phi} \otimes C(F) \stackrel{\Delta}{\rightarrow} C\left(\Sigma^{n}\right)_{\phi} \otimes C(F) \otimes C\left(\Sigma^{n}\right)_{\phi} \otimes C(F) \\
\psi \downarrow \downarrow \\
\qquad\left(\Sigma^{n} \times_{\tau} S(F)\right) \stackrel{\Delta}{\rightarrow} C\left(\Sigma^{n} \times_{\tau} S(F)\right) \otimes C\left(\Sigma^{n} \times_{\tau} S(F)\right)
\end{gathered}
$$

is commutative.

Define a map $h: \Sigma^{n} \rightarrow \Sigma^{n} \times{ }_{r} S(G)$ by $h(b)=b \times e_{0}, h\left(\sigma_{n}\right)=\sigma_{n} \times s_{0} \alpha^{-1}$. Using Lemma 7.2 , it is easily verified that $\psi$ is the composite

$$
\begin{aligned}
C\left(\Sigma^{n}\right)_{\phi} \otimes C(F) \stackrel{h_{*} \otimes 1}{\longrightarrow} C\left(\Sigma^{n} \times_{\tau} S(G)\right) \otimes C(F) \stackrel{\nabla}{\rightarrow} C\left(\Sigma^{n} \times_{\tau} S(G) \times S(F)\right) \\
\stackrel{(1 \times \mu)_{*}}{\longrightarrow} C\left(\Sigma^{n} \times_{\tau} S(F)\right) .
\end{aligned}
$$

Thus, the commutativity of (9.1) is equivalent to the commutativity of the following diagram.

$$
C\left(\Sigma^{n}\right)_{\phi} \otimes C(F) \stackrel{\Delta}{\longrightarrow} C\left(\Sigma^{n}\right)_{\phi} \otimes C(F) \otimes C\left(\Sigma^{n}\right)_{\phi} \otimes C(F)
$$

$$
\begin{aligned}
& C\left(\Sigma^{n} \times_{T} S(G)\right) \otimes C(F) \stackrel{\Delta}{\rightarrow} C\left(\Sigma^{n} \times_{T} S(G)\right) \otimes C(F) \otimes C\left(\Sigma^{n} \times_{T} S(G)\right) \otimes C(F) \\
& \nabla \downarrow \\
& C\left(\Sigma^{n} \times_{\tau} S(G) \times S(F)\right) \stackrel{\Delta}{\rightarrow} C\left(\Sigma^{n} \times_{\tau} S(G) \times S(F)\right) \otimes C\left(\Sigma^{n} \times_{\tau} S(G) \times S(F)\right) \\
& \begin{array}{ll}
\left(1 \times \mu_{*}\right) \downarrow & \downarrow(1 \times \mu)_{*} \otimes(1 \times \mu)_{*} \\
C\left(\Sigma^{n} \times_{\tau} S(F)\right) \longrightarrow \quad \Delta & \longrightarrow C\left(\Sigma^{n} \times_{\tau} S(F)\right) \otimes C\left(\Sigma^{n} \times_{\tau} S(F)\right) .
\end{array}
\end{aligned}
$$

The maps $\Delta$ in (9.2) denote the appropriate coproducts.

To prove $(9.2)$ commutes, it is clearly sufficient to prove that the three subdiagrams commute. The commutativity of the middle diagram is well known. (This is essentially the fact that the chain group of a topological group is a Hopf algebra.) The bottom diagram commutes because $1 \times \mu$ is a simplicial map. To prove that the top diagram commutes, note that $d_{0}\left(\sigma_{n} \times s_{0} \alpha^{-1}\right)=s_{0}^{n-1} b \times s_{0}^{n-1} e$, so for $c \in C_{p}(F)$,

$$
\begin{aligned}
\Delta\left(h_{*} \otimes 1\right)\left(\sigma_{n} \otimes c\right)= & \sum_{i=0}^{p} \sigma_{n} \times s_{0} \alpha^{-1} \otimes d_{i+1}^{p-i} c \otimes b \times e_{0} \otimes d_{0}^{i} c \\
& +\sum_{i=0}^{p}(-1)^{n i} b \times e \otimes d_{i+1}^{p-i} c \otimes \sigma_{n} \times s_{0}^{\alpha-1} \otimes d_{0}^{i} c \\
= & \left(h_{*} \otimes 1 \otimes h_{*} \otimes 1\right) \sum_{i=0}^{p}\left(\sigma_{n} \otimes d_{i+1}^{p-i} c \otimes b \otimes d_{0}^{i} c\right. \\
& \left.+(-1)^{n i} b \otimes d_{i+1}^{p-i} c \otimes \sigma_{n} \otimes d_{0}^{i} c\right)\left(h_{*} \otimes 1 \otimes h_{*} \otimes 1\right) \Delta\left(\sigma_{n} \otimes c\right) .
\end{aligned}
$$

This completes the proof of Theorem 8.2. 


\section{BiBLIOGRAPHY}

1. M. G. Barratt, V. K. A. M. Gugenheim, and J. C. Moore, On semisimplicial fiber bundles, Amer. J. Math. vol. 81 (1959) pp. 639-657.

2. E. G. Brown, On twisted tensor products. I, Ann. of Math. vol. 69 (1959) pp. 223-246.

3. V. K. A. M. Gugenheim, On a theorem of E. H. Brown, Illinois J. Math. vol. 4 (1960) pp. 292-311.

4. S. Eilenberg and S. MacLane, On the groups $H(\Pi, n)$. I, Ann. of Math. vol. 58 (1953) pp. 55-106.

5. - On the groups $H(I I, n)$. II, Ann. of Math. vol. 60 (1954) pp. 49-139.

6. M. Jean Leray, L'homologie d'un espace fibré dont la fibre est connexe, J. Math. Pures Appl. vol. 29 (1950) pp. 169-123. 396.

7. W. S. Massey, Exact couples in algebraic topology, Ann. of Math. vol. 56 (1952) pp. 363-

8. - On the cohomology ring of a sphere bundle, J. Math. Mech. vol. 7 (1958) pp. 265-290.

9. J. C. Moore, Seminar on algebraic homotopy theory, Princeton University (mimeographed).

10. N. Steenrod, The topology of fibre bundles, Princeton University Press, 1951.

University of Chicago,

Chicago, Illinois

DePaul. University,

Chicago, Illinois 